\title{
Distinct acoustic features in spinal and bulbar muscular atrophy patients with laryngospasm
}

4

5

Address correspondence to: Dr. Sobue, Department of Neurology, Nagoya University Graduate School of Medicine, 65 Tsurumai-cho, Showa-ku, Nagoya 466-8550, Japan

E-mail:sobueg@med.nagoya-u.ac.jp

Phone: +81-52-744-2385; Fax: +81-52-744-2384

Word count: main text, 2992 words; abstract, 215 words; title, 91 characters

No. of References, Tables, and Figures: 38 references, 6 tables, and 1 figure

Keywords: spinal and bulbar muscular atrophy, motor neuron, androgen receptor, laryngospasm, acoustic analysis, vocal folds. 
1 ABSTRACT

2 Objective: Laryngospasm is a sudden onset of transient respiratory difficulty that is

3 perceived as life-threatening by patients with spinal and bulbar muscular atrophy

4 (SBMA). The purpose of the study was to analyze the voice characteristics of SBMA

5 patients with laryngospasm using acoustic voice analysis.

6 Methods: Acoustic measurements were obtained from 39 consecutive Japanese

7 patients with genetically confirmed SBMA. A comparison was made between the 8 acoustic voice profiles of 16 patients with laryngospasm and 23 patients without

9 laryngospasm within 6 months before the evaluation. Computerized acoustic analysis

10 was performed for a prolonged vowel (/a:/) using the Multi-Dimensional Voice

11 Program (MDVP).

12 Results: SBMA patients with laryngospasm had smaller fluctuations of vocal fold

13 vibration and the turbulent noise component, indicating stronger vocal fold closure

14 than in those without laryngospasm. Receiver operating characteristic curve analysis

15 showed that the noise-to-harmonic ratio, which globally measures the noise 16 components of voice, is the most useful acoustic parameter to distinguish 17 laryngospasm (area under the curve $=0.767, p=0.007$ ).

18 Conclusions: The smaller noise component in patients with laryngospasm suggests

19 that the vocal folds of these patients are more adducted during phonation than those of

20 the patients without laryngospasm, even in the absence of laryngospasm. Quantitative

21 laryngeal analysis using the MDVP helps to detect laryngeal dysfunction and provides

22 physiological insight into the pathophysiology of laryngospasm in SBMA. 
1

2

3

4

5

6

7

\section{INTRODUCTION}

Spinal and bulbar muscular atrophy (SBMA), or Kennedy's disease, is a hereditary neurodegenerative disorder caused by the expansion of "CAG" repeats in the first exon of the androgen receptor (AR) gene. SBMA is classified as a lower motor neuron disease characterized by muscular atrophy, bulbar involvement, contraction fasciculations, and hand tremor. Patients occasionally demonstrate signs of androgen insensitivity such as gynecomastia [1-3]. Due to progressive bulbar muscular weakness, the majority of SBMA patients develop dysphagia and dysarthria [2-5].

Laryngeal involvement often occurs in subjects with SBMA, and it may lead to laryngospasm, which can be perceived as being life-threatening by patients $[6,7]$. Laryngospasm is defined as a sudden-onset episode with the sensation that air cannot be moved in and out of the lungs, which usually lasts for a few seconds and is followed by stridor, due to the rapid and forceful contraction of the laryngeal muscles [8], apparently resulting in close approximation of the arytenoid cartilages and vocal folds. In SBMA patients, laryngospasm can last from a few seconds to 1 minute. An SBMA patient who developed bilateral abductor vocal fold paralysis and respiratory distress, which was improved after tracheostomy, has also been reported [9]. Although it is clinically well-known that SBMA patients have a nasal voice [1], the voice characteristics of SBMA patients have not been evaluated using perceptual and acoustical analyses. It is clinically important to investigate the laryngeal function of SBMA patients in detail since this symptom is perceived as life-threatening by all SBMA patients with laryngospasm [6,10].

In the assessment of laryngeal function during phonation, perceptual and acoustical measures provide substantial information. Acoustic analysis has been used in Parkinson's disease and amyotrophic lateral sclerosis (ALS) to quantify 
1 characteristics of voice production, which cannot be conceived by auditory perceptual

2 impression [11,12]. Particularly, Multi-Dimensional Voice Program (MDVP) analyses

3 have been applied extensively for voice analysis of patients with dysarthria [13], and

$4 \quad$ will be used here.

$5 \quad$ The purpose of this study was to perform perceptual assessment and acoustic analysis to clarify the differences in the voice characteristics of SBMA patients with and without laryngospasm.

\section{METHODS}

Ethics. This study was approved by the ethics committees of Aichi-Gakuin University School of Health Science and Nagoya University Graduate School of Medicine. All participants gave their written informed consent prior to the interviews and examinations.

Subjects. Thirty-nine SBMA patients genetically diagnosed as having CAG repeat expansion $(\geq 38)$ in the first exon of the AR gene were included in this study [14]. No patients had definite symptoms or took medication for gastroesophageal reflux disease, although we did not perform endoscopic examinations. No subjects had tracheostomy or underwent recent intubation for any surgery. Age of onset was defined as the age at which the patient became aware of muscular weakness in any part of the body, including bulbar symptoms. The patients were divided into 2 groups according to their history of laryngospasm, i.e., patients who experienced laryngospasm within 6 months before the acoustic examination and those without such an episode. The frequency and trigger factors of laryngospasm and disease severity were evaluated in each patient through history taking (Table 1). All SBMA patients were subjected to perceptual assessments and MDVP analysis to assess current laryngeal function. 
1 However, 2 patients who had difficulty in vocalization and breathing during voice

2 recording of the prolonged vowel /a:/ (Patients no. 1 and 7) were excluded from the

3 MDVP analysis.

4

5 Comprehensive disease severity scales. The disease severity of each SBMA patient

6 was assessed using functional scales such as the amyotrophic lateral sclerosis

7 functional rating scale-revised (ALSFRS-R), Limb Norris score, and Norris Bulbar score $[15,16]$. These scales have been used for SBMA and are strongly correlated with each other $[17,18]$.

11 Genetic analysis. Genetic analysis was done to confirm the diagnosis of SBMA.

Genomic DNA was extracted from the peripheral blood of SBMA patients using conventional techniques. PCR amplification of the CAG repeat in the AR gene was performed using a fluorescein-labeled forward primer (5'-TCCAGAATCTGTTCCAGAAATGC-3') and a non-labeled reverse primer (5'-TGGCCTCGCTCAGGATGTCTTTAAG-3'). The detailed PCR method has been described previously [19]. Aliquots of the PCR products were combined with a loading dye and separated by electrophoresis using an autoread sequencer (SQ-5500; Hitachi Electronics Engineering, Tokyo, Japan). The CAG repeat size of the PCR standards was determined by direct sequencing, as described previously [19].

\section{Auditory perceptual voice assessments}

The speech of each SBMA patient included in the present study was evaluated

24 perceptually to define their voice characteristics.

25 GRBAS analysis. Perceptual voice analysis for dysphonia was performed using the 
1 Grade, Roughness, Breathiness, Asthenia, and Strain (GRBAS) scale, in which the

2 voice properties were evaluated with respect to dysphonia: Grade $(G)$, the overall

3 degree of the deviance of voice; Roughness (R), irregular fluctuation of the

4 fundamental frequency; Breathiness (B), turbulent noise produced by air leakage;

5 Asthenia (A), overall weakness of the voice; and Strain (S), impression of tenseness

6 or excess effort. Each component was scored using a four-point scale: 0, normal; 1,

7 mild deviance; 2, moderate deviance; and 3, severe deviance [20-22].

8 Hypernasality. Perceptual assessment of hypernasality was performed according to a

9 previous study [23]. Speech sound was scored using a four-point scale: 1, normal; 2,

10 mild presence of hypernasality; 3, moderate presence of hypernasality; and 4, severe 11 presence of hypernasality.

12 Procedures of perceptual assessments. Three types of speech samples (the prolonged 13 vowel /a:/, reading the passage "The North Wind and the Sun," and conversation) were used for the perceptual assessments. Each speech sample, which had been recorded in a randomized order, was rated independently by two judges (certified speech-language pathologists) using the above dimensions. The judges had an unlimited time to listen to and rate the speech samples. The measurement values were calculated from the average score of the two judges. After the independent rating of the speech samples, the two judges conferred to reach an agreement on each speech dimension [23]. Before the consensus rating, the ratings of the judges did not vary by more than 1 point.

\section{Acoustic analysis}

Recording procedures and signal processing. Voice recordings were performed at a sampling frequency of $44,100 \mathrm{~Hz}$ and a resolution of 16 bits using a liner PCM 
1 recorder (R-09HR; Roland Corporation, Shizuoka, Japan) and a condenser

2 microphone (ECM-23F5; Sony, Tokyo, Japan) in a quiet room with a noise level

3 below $35 \mathrm{~dB}$. The distance from the microphone to the mouth was $15 \mathrm{~cm}$.

4 The subjects were asked to phonate a sustained /a:/ vowel for approximately $5 \mathrm{~s}$ at a

5 comfortable loudness and constant pitch [13]. This task was performed twice.

$6 \quad M D V P$. Computerized acoustic analyses of the sustained vowel were performed using

7 MDVP Model 4300 (Kay Electrometrics, Lincoln Park, NJ, USA). Voice was analyzed for $3 \mathrm{~s}$ in the mid-portion of the sustained vowel, excluding the first and last

$925 \mathrm{~ms}$ of the vowel according to a previous report [13].

We calculated 33 out of 34 parameters of MDVP, most of which have been validated with regard to the reliability in previous studies of other neurological diseases $[11,13,24]$. Values lying outside the normal range of these parameters suggest dysphonia (mostly audible as hoarseness) [24-26]. We did not use Tsam, the parameter which evaluate the time length of voice samples, since we analyzed 3-second voice samples for each patient in the present study.

The measurement values were calculated from an average of two consecutive phonations, as described previously [13]. All MDVP measurements were performed at Nagoya University Hospital.

Statistical analysis. We analyzed the data using the non-parametric Mann-Whitney test for statistical comparisons. Correlations among the parameters were analyzed using Spearman's rank correlation coefficient. To investigate the reliability of the acoustic analysis, the intra-class correlation coefficient (ICC) was calculated between the two samples. A receiver operating characteristic (ROC) curve, which is obtained by plotting the sensitivity of a test on the $y$-axis against 1-specificity on the $\mathrm{x}$-axis, 
1 was used to determine the validity of the screening test. The area under the curve

2 (AUC), a measure of the accuracy of the screening tool calculated from the ROC

3 curve, was applied for the detection of laryngospasm. The optimal cut-off point was

4 determined on the point of the ROC curve closest to the upper left-hand corner $(0,1)$

5 [27]. $P$-values below 0.05 were considered to be statistically significant. Calculations

6 were performed using the statistical software package IBM SPSS ver.19.

7

\section{RESULTS}

The 39 male Japanese patients with SBMA were analyzed consecutively, as shown in Tables 1 and 2. Among the patients, 16 (41.0\%) had a recent episode of laryngospasm. The clinical and genetic backgrounds, such as age, number of CAG repeats, disease duration, testosterone levels, and disease severity, were equivalent between the SBMA patients with and without laryngospasm (Table 2).

The most common trigger of laryngospasm in the SBMA patients was aspiration (12 patients/16 patients, $75.0 \%$ ), followed by phonation in 4/16 patients $(25.0 \%)$. Laryngospasm also occurred spontaneously (without subjective triggers) in 2/16 patients $(12.5 \%)$. As for frequency, laryngospasm occurred more than once per day in 2 patients $(2 / 16,12.5 \%)$, more than once per week in 1 patient $(1 / 16,6.3 \%)$, more than once per month in 6 patients $(6 / 16,37.5 \%)$, and more than once per 6 months in 7 patients $(7 / 16,43.8 \%)$ during the 6 months before the acoustic evaluation.

Perceptual features of the SBMA patients with laryngospasm. Table 3 shows the auditory perceptual parameters for hoarseness and hypernasality in the SBMA patients. In the present study, 28 of the $39(71.2 \%)$ patients were confirmed to have dysphonia by perceptual voice analysis with GRBAS. In the majority of SBMA patients with 
1 dysphonia, the severity of dysphonia was mild (G: 0.5-1). There was no significant

2 difference in the perpetual assessment values between the SBMA patients with and

3 without laryngospasm.

4

5 Acoustic features of the SBMA patients with laryngospasm. Table 4 shows the comparisons of the MDVP parameters between the SBMA patients with and without laryngospasm. Overall, the differences between the groups were predominantly observed in the parameters regarding frequency perturbation rather than those related to amplitude perturbation. The patients with laryngospasm showed decreased values of the indices regarding frequency perturbation, such as Jitt. Noise-related indices, VTI and NHR, were also altered in the SBMA patients with laryngospasm. As for amplitude perturbation, although Shim was decreased in the patients with laryngospasm, other parameters showed no differences between the groups.

The 7 parameters (Jita, Jitt, APQ, PPQ, Shim, VTI and NHR), which showed a significant difference in the comparison of the two groups, showed a strong intra-class correlation between the duplicated samples, except for Jitt; Jita, ICC $=0.82(95 \%$ $\mathrm{CI}=0.67-0.90, \quad p<0.001) ; \quad \mathrm{Jitt}, \quad \mathrm{ICC}=0.57 \quad(95 \% \quad \mathrm{CI}=0.18-0.77, \quad p=0.005) ;$ Shim: $\mathrm{ICC}=0.81 \quad(95 \% \quad \mathrm{CI}=0.64-0.90, \quad p<0.001) ; \mathrm{APQ}, \quad \mathrm{ICC}=0.85 \quad(95 \% \quad \mathrm{CI}=0.72-0.92$, $p=0.005) ; \mathrm{PPQ}, \mathrm{ICC}=0.84 \quad(95 \% \mathrm{CI}=0.69-0.92, p=0.005) ; \mathrm{VTI}, \mathrm{ICC}=0.71 \quad(95 \%$ $\mathrm{CI}=0.47-0.84, p=0.001)$; and NHR, ICC=0.83 (95\% CI=0.70-0.91, $p<0.001)$. These results indicate the high reliability of our acoustic assessments. From these parameters, we selected the following indices for further analyses: (1) frequency perturbation of vocal fold vibration (Jita: absolute Jitter, and Jitt: jitter percent): when dysfunction of the intrinsic laryngeal muscles occurs, fundamental frequency may become less stable, causing a rise in Jita and Jitt; (2) amplitude perturbation of vocal fold vibration (Shim: 
1 shimmer percent): when dysfunction of the intrinsic laryngeal muscles and respiratory

2 muscles occurs, cycle amplitudes may become less stable, causing a rise in Shim; (3)

3 turbulent noise (VTI: voice turbulence index): when vocal fold closure is incomplete,

4 the turbulent noise produced by the aperture between the vocal folds may increase,

5 causing a rise in VTI; and (4) various noises-related index (NHR: noise-to-harmonic

6 ratio): an increase in any of the noise components, i.e., Jita, Jitt, ShdB, Shim, or VTI,

7 may lead to elevated NHR [11-13,25,28]. We did not use RAP or PPQ for the detailed

8 analysis, as the clinical implication of these indices is less established than that of the

9 other parameters [24].

10 As for the relationships between the MDVP parameters and clinical background, 11 Jitt, Shim, and NHR were inversely and mildly correlated with the frequency of 12 laryngospasm ( $r=-0.346, p=0.036 ; \quad r=-0.328, p=0.047$; and $r=-0.460, p=0.004$; 13 respectively). Shim was also inversely and mildly correlated with Limb Norris score 14 ( $r=-0.447, p=0.022)$. There was no clinically relevant correlation between clinical 15 background and the other MDVP parameters (Table 5).

16 ROC curve analysis was applied between the SBMA patients with and without 17 laryngospasm. The results demonstrated that Jita, Jitt, Shim and NHR were significantly lower in the patients with laryngospasm than in those without (Table 6

19 and Figure 1). The AUC data suggest that NHR was the acoustic parameter most strongly associated with laryngospasm in SBMA patients.

\section{DISCUSSION}

23 In the present study, although perceptual assessments did not show a significant 24 difference between the SBMA patients with laryngospasm and those without laryngospasm, quantitative acoustic analysis identified the acoustic characteristics of 
1 voice production in SBMA patients with laryngospasm. Our results suggest that NHR,

2 which evaluates various noises due to frequency and amplitude perturbation, turbulent

3 noise and/or voice breaks during phonation, appears to be a useful acoustic parameter

4 for distinguishing the SBMA patients with laryngospasm from those without

5 laryngospasm.

6 This study is the first to investigate the voice characteristics in individuals with

7 SBMA. A laryngeal fiberscope can be used to visualize the larynx for any vocal fold

8 pathology, but the procedure can trigger laryngospasm [29]. Conversely, acoustic

9 analysis, particularly the MDVP used in the present study, is a quantitative assessment

10 tool for patients, and has a low risk of inducing laryngospasm by virtue of its non-invasive nature. The absence of a strong relationship between the acoustic parameters and the ALSFRS-R and Norris scales suggests that laryngeal dysfunction in SBMA cannot be evaluated precisely using motor function scales, but that acoustic measurements appear to be helpful in assessing vocal function in this disorder.

SBMA patients with laryngospasm, compared with those without laryngospasm, showed significantly smaller values in frequency perturbation indices, such as Jitt, and noise-related indices, such as VTI and NHR, during phonation. In consideration of its correlation coefficient and ROC curve, NHR is useful to understand the mechanism of laryngospasm in SBMA patients. As for frequency perturbation indices, abnormal increases of these parameters are explained by several factors, including a reduction in the number of motor neurons, decreased mean firing rate of the motor units of the laryngeal muscles, and variation in the size of motor units and action potentials [30]. Some of these factors are likely present in the limbs of SBMA patients [18]. Abnormalities in the frequency and amplitude of voice have been shown to be related to lesions of the nucleus ambiguous [31]. The low values of frequency and 
1 amplitude perturbation indices in the SBMA patients with laryngospasm suggest the

2 relatively mild loss of lower motor neurons innervating the laryngeal muscles

3 compared to those without laryngospasm.

The degree of vocal fold closure is determined by a balance of forces between the vocal fold abductor and adductor muscles [32,33]. Either the decreased activity of the abductor muscle or increased activity of the adductor muscles results in the stronger closure of the vocal folds [29]. Given that VTI may be related to the size of the aperture between the vocal folds during phonation, our finding that SBMA patients with laryngospasm had lower VT1 values than those without laryngospasm suggests the greater closure of the vocal folds, which is indicative of an imbalance of force between the vocal fold abductor and adductor muscles during phonation. This hypothesis is supported by the observation that a bilateral reduction in the amplitude of the abduction of the vocal folds may occur in SBMA patients [9,34]. Histological examination of the intrinsic laryngeal muscles of such patients revealed severe neurogenic changes in the abductor muscle rather than in the adductor muscles [35].

The main causes of the noise used to calculate the NHR values are incomplete glottal closure (VTI) and voice signal instability, such as frequency and amplitude perturbation [24]. Increased NHR values are consistent with increased Jita, Jitt, Shim, or VTI values. Therefore, our finding that NHR is the most useful acoustic parameter for laryngospasm agrees with the changes of both important parameters in SBMA patients with laryngospasm, i.e., low values of frequency perturbation indices indicate milder neuronal loss, and a low VTI value suggests an imbalance of force between the abductor and adductor muscles.

In the majority of patients with SBMA in our study, laryngospasm was observed after a trigger, such as aspiration or phonation. It is suggested that obvious triggers 
1 leading to strong vocal fold closure are required for laryngospasm to occur. In an

2 experimental study using animals, increased excitability of the motor neurons

3 innervating the laryngeal adductor muscles was detected during and after the cough

4 reflex [36]. The results of the present study suggest that the vocal folds are more

5 adducted during phonation, even in the absence of laryngospasm, in SBMA patients

6 with laryngospasm. Additional stimuli, such as the cough reflex to expel aspirated

7 materials, may further intensify the spastic closure of the vocal folds, which is

8 sustained because of a limitation in the range of the opening movement of the vocal

9 folds, resulting from an imbalance of force between the abductor and adductor

10 muscles. These findings are likely to represent one of the mechanisms underlying

11 laryngospasm in SBMA. In the present study, 16 of the 39 patients $(41.0 \%)$ with

12 SBMA had a recent episode of laryngospasm. Agreeing with our data, laryngospasm

13 reportedly occurs in $47 \%$ of SBMA patients, while it only occurs in $2 \%$ of ALS

14 patients [6], although the clinical motor neuron symptoms of SBMA overlap with

15 those of ALS [37]. Severe adductor muscle dysfunction is reported in ALS patients

16 [38], suggesting that ALS patients have difficulty in completely closing their vocal

17 folds due to the hypoactivity of their adductor muscles compared with SBMA

18 patients.

19 In conclusion, we applied the MDVP, which is a simple, objective, quantitative, and non-invasive tool, to assess voice disorders in SBMA patients. Our results suggest the

21 hypothesis that laryngospasm may occur in patients who develop excessive vocal fold

22 closure due to the imbalance of force between the abductor and adductor muscles.

23 Quantitative laryngeal analysis using the MDVP appears to provide mechanistic

24 insight into the pathophysiology of laryngospasm in SBMA patients. 


\section{About disclosure}

5 The authors have no conflict of interests. Dr. Katsuno is supported by a Grant-in-Aid

6 for Scientific Research on Innovated Areas "Foundation of Synapse and Neurocircuit

7 Pathology" from MEXT, Japan (22110005); KAKENHI grants from MEXT/JSPS, 8 Japan (No. 21229011, 23390230); and Core Research for Evolutional Science and 9 Technology (CREST) from the Japan Science and Technology Agency (JST). Dr. 10 Adachi is funded by the Ministry of Education, Culture, Sports, Science and 11 Technology of Japan and JST. Dr. Sobue serves on the scientific advisory board of the 12 Kanae Science Foundation for the Promotion of Medical Science and Naito Science 13 Foundation; serves as an advisory board member of Brain, an editorial board member of Degenerative Neurological and Neuromuscular disease, the Journal of Neurology, and Amyotrophic Lateral Sclerosis and Frontotemporal Degeneration; and is funded by the Ministry of Education, Culture, Sports, Science and Technology of Japan, the Ministry of Welfare, Health and Labor of Japan, and CREST, JST. The other authors report no disclosure.

\section{Individual contributions to the manuscript}

Mr. Tanaka made the study concept and design, analysis and interpretation of data, contribution of vital tools, acquisition of data, and statistical analysis.

Dr. Banno made the study concept and design, drafting and revising the manuscript, analysis and interpretation of data, acquisition of data, and statistical analysis.

Dr. Katsuno made the study concept and design, drafting and revising the manuscript, analysis and interpretation of data, acquisition of data, and statistical analysis. 
1 Dr. Suzuki made the study concept and acquisition of data.

2 Dr. Suga made the study concept and acquisition of data.

3 Dr. Hashizume made the study concept and acquisition of data.

4 Dr. Mano made the study concept and acquisition of data.

5 Dr. Araki made the study concept and acquisition of data.

6 Dr. Watanabe made the study concept and acquisition of data.

$7 \quad$ Dr. Adachi made the study concept and acquisition of data.

8 Mr. Tatsumi made the study concept.

9 Dr. Yamamoto made the study concept and design, drafting and revising the 10 manuscript, analysis and interpretation of data, and study supervision.

11 Dr. Sobue made the study concept, drafting and revising the manuscript, analysis and 12 interpretation of data, and study supervision. 


\section{References}

1. Kennedy WR, Alter M, Sung JH. Progressive proximal spinal and bulbar muscular atrophy of late onset. A sex-linked recessive trait. Neurology 1968;18:671-80.

2. Sobue G, Hashizume Y, Mukai E, Hirayama M, Mitsuma T, Takahashi A. X-linked recessive bulbospinalneuronopathy. A clinicopathological study. Brain 1989;112: 209-32.

3. Katsuno M, Tanaka F, Adachi H. Pathogenesis and therapy of spinal and bulbar muscular atrophy (SBMA). Progress in Neurobiology 2012;99:246-56.

4. Atsuta N, Watanabe H, Ito M, Banno H, Suzuki S, Katsuno M, et al. Natural history of spinal and bulbar muscular atrophy (SBMA): a study of 223 Japanese patients. Brain 2006;129:1446-55.

5. Banno H, Katsuno M, Suzuki K, Takeuchi Y, Kawashima M, Suga N, et al. Phase 2 trial of leuprolin in patients with spinal and bulbar muscular atrophy. Ann Neurol 2009;65:140-50.

6. Sperfeld AD, Hanemann CO, Ludolph AC, Kassubek J. Laryngospasm: An underdiagnosed symptom of X-linked spinobulbar muscular atrophy. Neurology 2005;64:753-4.

7. Niesen AD, Sprung J, Prakash YS, Watson JC, Weingarten TN. Case series: anesthetic management of patients with spinal and bulbar muscular atrophy (Kennedy’s disease). Can J Anesth 2009;56:136-44.

8. Maceri DR, Zim S. Laryngospasm: an atypical manifestation of sever gastroesophageal reflex disease (GERD). Laryngoscopy 2001;111:1976-9.

9. Tomiyasu K, Saito T, Nukazawa T, Ito H, Kowa H. A case of X-linked bulbospinal muscular atrophy with bilateral abductor vocal cord paralysis. Clin Neurol 1996;36:683-6. 
1 10. Blitzer A, Brin MF, Raming LO. Neurologic disorders of the larynx, 2th. New

2 York: Thieme Medical Publishers, 2009:217-8.

3 11. Midi I, Dogan M, Koseoglu M, Can G, Sehitoglu MA, Gunal DI. Voice 4 abnormalities dysfunction and their relation with motor dysfunction in Parkinson's disease. Acta Neurol Scand 2008;117:26-34.

12. Silbergleit AK, Johnson AF, Jacobson BH. Acoustic analysis of voice in individuals with amyotrophic lateral sclerosis and perceptually normal voice quality. J Voice 1997;11:222-31.

13. Kent RD, Vorperian HK, Kent JF, Duffy JR. Voice dysfunction in dysarthria: application of the multi-dimensional voice $\operatorname{program}^{\mathrm{TM}}$. J Commun Disord 2003;36:281-306.

14. La Spada AR, Wilson EM, Lubahn DB, Harding AE, Fischbeck KH. Androgen receptor gene mutations in X-linked spinal and bulbar muscular atrophy. Nature $1991 ; 352: 77-9$.

15. The ALS CNTF Treatment Study (ACTS) Phase I - II Study Group. The amyotrophic lateral sclerosis function rating scale: Assessment of activities of daily in patients with ALS. Arch Neurol 1996;53:141-7.

16. Norris FH, Calanchini PR, Fallat RJ, Panchari S, Jewett B. The administration of guanidine in amyotrophic lateral sclerosis. Neurology 1974;24:721-8.

17. Katsuno M, Banno H, Suzuki K, Takeuchi Y, Kawashima M, Yabe I, et al. Efficacy and safety of leuprorelin in patients with spinal and bulbar muscular atrophy (JASMITT study): a multicenter, randomized, double-blind, placebo-controlled trial. Lancet Neurol 2010;9:875-84.

18. Suzuki K, Katsuno M, Banno H, Takeuchi Y, Kawashima M, Suga N, et al. The profile of motor unit number estimation (MUNE) in spinal and bulbar muscular 
atrophy. J Neurol Neurosurg Psychiatry 2010;81:567-71.

19. Doyu M, Sobue G, Mukai E, Kachi T, Yasuda T, Mitsuma T, et al. Severity of X-linked recessive bulbospinal neuronopathy correlates with size of the tandem CAG repeat in androgen receptor. Ann Neurol 1992;32:707-10.

20. Dejonekere PH, Remacle M, Frenel-Elbaz E, Woisard V, Creview-Buchman L, Millet B. Differentiated perceptual evaluation of pathological voice quality: reliability and correlation with acoustic measurements. Rev Laryngol Otol Rhinol 1996;117:219-24.

21. Carding PN, Docherty G, Horsley IA. A study of the effectiveness of voice therapy in the treatment of forty-five patients with non-organic dysphonia. $\mathrm{J}$ voice 1999;13:76-113.

22. Nemr K, Simose-Zenari M, Corderio GF, Tsuji D, Ogawa AI, Ubrig MT, et al. GRBAS and Cape-V scales: high reliability and consensus when applied at different times. J Voice 2012;26:17-22.

23. Vitorino J. Velopharyngeal function in adult speakers of Portuguese diagnosis with multiple sclerosis. NeuroRehabilitation 2009;25:279-87.

24. Blitzer A, Brin MF, Raming LO. Neurologic disorders of the larynx, 2th. New York: Thieme Medical Publishers, 2009:74-84.

25. Johnson AF, Jacobson BH. Medical speech-language pathology, a practitioner's guide, 2th. New York: Thieme Medical Publishers, 2006:161-3.

26. Mori K, Kojima H, Haji T, Honjo I. Voice characteristics of aged persons. Pract Otorhinolaryngol 1988;81:737-54.

27. Akobeng AK. Understanding diagnostic test 3: receiver operating characteristic curves. Acta Paediatr 2007;96:644-7.

28. Robert D, Pouget J, Giovanni A, Azulay JP, Triglia JM. Quantitative voice 
analysis in the assessment of bulbar involvement in amyotrophic lateral sclerosis. Acta Otolaryngol (Stockh) 1999;119:724-31.

29. Graaff MM, Grolam W, Westermann EJ, Boogaardt HC, Koelman H, Kooi AJ, et al. Vocal cord dysfunction in Amyotrophic Lateral Sclerosis. Arch Neurol 2009;66:1329-33.

30. Titze IR. A model for neurologic sources of aperiodicity in vocal fold vibration. J Speech Hear Res 1991;34:460-72.

31. Raming LA, Scherer RC, Titze IR, Ringel SP. Acoustic analysis of voices of patients with neurologic disease: rationale and preliminary data. Ann Otol Rhinol Laryngol 1988;97:164-72.

32. Ludlow CL. Central nervous system control of the laryngeal muscles in humans. Respir Physiol Neurobiol 2005;147:205-22.

33. Hillel AD. The study of laryngeal muscle activity in normal human subjects and in patients with laryngeal dystonia using multiple fine-wire electrography. Laryngoscope 2001;111:1-47.

34. Warnecke T, Oelenberg S, Teismann I, Suntrup S, Hamacher C, Young P, et al. Dysphasia in X-linked bulbospinal muscular atrophy (Kennedy disease). Neuromuscul Disord 2009;19:704-8.

35. Mochizuki Y, Mochizuki T, Hirose H, Nishiyama K, Tsukuda M. A case of spinal and bulbar muscular atrophy (Kennedy-Alter-Sung Disease) with dysphagia treated by total laryngectomy. J Jpn Bronchesopagol Soc 2009;60:35-40.

36. Katada A, Enomoto K, Kobayashi Y, Nonaka S, Unno T, Sakamoto T. Changes in laryngeal adductor activities after the cough reflex in cats. J Jpn Bronchoesophagol Soc 1996;47:340-5.

37. Paparounas K, Gotsi A, Syrrou M, Akritidis N. Kennedy disease: avoiding 
1 misdiagnosis. Arch Neurol 2003;60:893-4.

2 38. Isozaki E, Hayashi M, Hayashida T, Oda M, Hirai S. Myopathology of the 3 intrinsic muscles in neurodegenerative diseases, with reference to the mechanism $4 \quad$ of vocal cord paralysis. Clin Neurol 1998;38:711-8. 


\section{$1 \quad$ Figure legends}

2

3 Figure 1. Receiver operating characteristic curve analysis in the SBMA patients

4 with and without laryngospasm

5 ROC curve analysis of SBMA patients with and without laryngospasm using (A) Jita,

6 (B) Jitt, (C) Shim, (D) VTI, and (E) NHR is shown. The curve is obtained by

7 calculating the sensitivity and specificity of the test at every possible cut-off point,

8 and plotting sensitivity against 1-specificity. Sensitivity (the proportion of true

9 positive results) is shown on the y-axis, going from 0 to 1 (0-100\%), and 1-specificity

10 (the proportion of false positive results) is shown on the $\mathrm{x}$-axis, going from 0 to 1

$11(0-100 \%)$. The best cut-off point to balance the sensitivity and specificity of a test is

12 the one closest to the $(0,1)$ point. The two circles show each statistically significant

13 optimal cut-off point, as demonstrated in Table 6.

14 Abbreviations: Jita, absolute jitter; Jitt, jitter percent; Shim, shimmer percent; VTI,

15 voice turbulence index; NHR, noise-to-harmonic ratio. 
1 Table 1. Profiles of the SBMA patients

\begin{tabular}{|c|c|c|c|c|c|c|c|c|c|c|}
\hline \multirow[b]{2}{*}{$\begin{array}{l}\text { Patient } \\
\text { no. }\end{array}$} & \multirow{2}{*}{$\begin{array}{l}\text { Frequency of } \\
\text { laryngospasm } \\
\text { during } 6 \text { months } \\
\text { before evaluation }\end{array}$} & \multirow{2}{*}{$\begin{array}{l}\text { Age at } \\
\text { evaluation, } \\
\text { years }\end{array}$} & \multirow{2}{*}{$\begin{array}{l}\text { Age at } \\
\text { onset, } \\
\text { years }\end{array}$} & \multirow{2}{*}{$\begin{array}{l}\text { Disease } \\
\text { duration } \\
\text { from onset }{ }^{\mathrm{e}} \\
\text { years }\end{array}$} & \multirow{2}{*}{$\begin{array}{l}\text { CAG-repeat } \\
\text { length } \\
\text { in the AR } \\
\text { gene, } \\
\text { number }\end{array}$} & \multirow{2}{*}{$\begin{array}{l}\text { Testosterone, } \\
\text { ng/dL }\end{array}$} & \multicolumn{2}{|l|}{ ALSFRS-R } & \multicolumn{2}{|c|}{ Norris Scale } \\
\hline & & & & & & & $\begin{array}{l}\text { Bulbar } \\
\text { subscore }^{\mathrm{d}}\end{array}$ & Total & LNS & NBS \\
\hline 1 & ++++ & 58 & 36 & 22 & 60 & 4.59 & 8 & 37 & 55 & 21 \\
\hline 2 & ++++ & 68 & 35 & 33 & 48 & 5.69 & 8 & 34 & $\mathrm{NA}$ & $\mathrm{NA}$ \\
\hline 3 & +++ & 63 & 36 & 27 & 53 & 4.65 & 9 & 36 & $\mathrm{NA}$ & $\mathrm{NA}$ \\
\hline 4 & ++ & 58 & 55 & 3 & 54 & 5.61 & 12 & 48 & 62 & 39 \\
\hline 5 & ++ & 62 & 50 & 12 & 51 & 6.75 & 10 & 42 & 59 & 33 \\
\hline 6 & ++ & 72 & 67 & 5 & 43 & 4.95 & 10 & 41 & 52 & 31 \\
\hline 7 & ++ & 58 & 48 & 10 & 45 & 3.75 & 8 & 35 & 39 & 26 \\
\hline 8 & ++ & 58 & 49 & 9 & $\mathrm{NA}$ & 7.68 & 10 & 38 & $\mathrm{NA}$ & $\mathrm{NA}$ \\
\hline 9 & ++ & 60 & 45 & 15 & $\mathrm{NA}$ & 3.83 & 8 & 34 & $\mathrm{NA}$ & $\mathrm{NA}$ \\
\hline 10 & + & 29 & 23 & 6 & 53 & 10.85 & 11 & 45 & 60 & 39 \\
\hline 11 & + & 43 & 33 & 10 & 48 & 9.53 & 11 & 45 & 60 & 38 \\
\hline 12 & + & 63 & 44 & 19 & 48 & 7.07 & 9 & 37 & 46 & 33 \\
\hline 13 & + & 71 & 66 & 5 & 43 & 6.42 & 11 & 43 & 53 & 30 \\
\hline 14 & + & 50 & 30 & 20 & 46 & 5.13 & 10 & 36 & 41 & 24 \\
\hline 15 & + & 63 & 59 & 4 & 46 & 6.67 & 9 & 38 & 48 & 28 \\
\hline 16 & + & 53 & 38 & 15 & $\mathrm{NA}$ & 7.92 & 9 & 41 & $\mathrm{NA}$ & $\mathrm{NA}$ \\
\hline 17 & - & 40 & 37 & 3 & 50 & 12.02 & 12 & 44 & 59 & 38 \\
\hline 18 & - & 48 & 46 & 2 & 47 & 10.29 & 10 & 44 & 57 & 34 \\
\hline 19 & - & 40 & 34 & 6 & 49 & 8.04 & 12 & 48 & 58 & 37 \\
\hline 20 & - & 73 & 14 & 59 & 46 & 8.01 & 11 & 45 & 60 & 38 \\
\hline 21 & - & 59 & 50 & 9 & 51 & 4.78 & 12 & 43 & 54 & 37 \\
\hline 22 & - & 39 & 22 & 17 & 52 & 4.55 & 11 & 44 & 62 & 38 \\
\hline 23 & - & 37 & 30 & 7 & 54 & 4.54 & 12 & 45 & 59 & 37 \\
\hline 24 & - & 72 & 60 & 12 & 45 & 4.78 & 11 & 42 & 56 & 36 \\
\hline 25 & - & 50 & 30 & 20 & 49 & 4.60 & 10 & 43 & 61 & 36 \\
\hline 26 & - & 69 & 54 & 15 & 49 & 5.47 & 11 & 39 & 43 & 34 \\
\hline 27 & - & 48 & 38 & 10 & 45 & 9.44 & 11 & 41 & 61 & 28 \\
\hline 28 & - & 73 & 69 & 4 & 40 & 6.46 & 11 & 43 & 58 & 32 \\
\hline 29 & - & 61 & 55 & 6 & 46 & 10.03 & 12 & 43 & 49 & 32 \\
\hline 30 & - & 47 & 38 & 9 & 50 & 13.11 & 10 & 39 & 51 & 26 \\
\hline 31 & - & 54 & 40 & 14 & 51 & 6.82 & 12 & 41 & 53 & 32 \\
\hline 32 & - & 47 & 38 & 9 & 50 & 4.31 & 10 & 42 & 53 & 28 \\
\hline 33 & - & 77 & 63 & 14 & 46 & 5.80 & 5 & 26 & 27 & 21 \\
\hline 34 & - & 56 & 40 & 16 & 51 & 4.55 & 10 & 39 & 50 & 30 \\
\hline 35 & - & 58 & 48 & 10 & 45 & 4.53 & 8 & 37 & 45 & 27 \\
\hline 36 & - & 55 & 48 & 7 & $\mathrm{NA}$ & 11.94 & 8 & 31 & $\mathrm{NA}$ & $\mathrm{NA}$ \\
\hline 37 & - & 61 & 49 & 12 & $\mathrm{NA}$ & 8.54 & 10 & 40 & $\mathrm{NA}$ & $\mathrm{NA}$ \\
\hline 38 & - & 58 & 39 & 19 & 48 & 6.99 & 10 & 37 & $\mathrm{NA}$ & $\mathrm{NA}$ \\
\hline 39 & - & 70 & 55 & 15 & $\mathrm{NA}$ & 5.96 & 8 & 35 & $\mathrm{NA}$ & $\mathrm{NA}$ \\
\hline
\end{tabular}

2 Abbreviations: NA, not available; ALSFRS-R, revised amyotrophic lateral sclerosis

3 functional rating scale; LNS, Limb Norris score; NBS, Norris Bulbar score. 
$1 \quad{ }^{\mathrm{a}}$ Frequency of laryngospasm during the 6 months before the evaluation: ++++ , more

2 than once per day; +++ , more than once per week; ++ , more than once per month; + ,

3 more than once per 6 months; and -, without laryngospasm.

4 bge at onset was defined as the age at which a patient became aware of muscular

$5 \quad$ weakness in any part of the body, including bulbar symptoms.

$6 \quad{ }^{\mathrm{c}}$ Disease duration from onset was defined as the period of time between the age at

$7 \quad$ onset and evaluation.

$8{ }^{\mathrm{d}}$ The bulbar subscore was calculated as the total number of items related to bulbar

9 signs consisting of speech, salivation, and swallowing. 
1 Table 2. Clinical and genetic features of the 39 SBMA patients with and without

2 laryngospasm

\begin{tabular}{|c|c|c|c|c|}
\hline \multirow{3}{*}{ Demographic } & \multicolumn{4}{|c|}{ SBMA patients ( $n=39$ ) } \\
\hline & $\begin{array}{l}\text { All } \\
(n=39)\end{array}$ & $\begin{array}{l}\text { Without } \\
\text { laryngospasm } \\
(\mathbf{n = 2 3})\end{array}$ & $\begin{array}{l}\text { With } \\
\text { laryngospasm } \\
(n=16)\end{array}$ & $\begin{array}{l}\text { With vs. } \\
\text { without } \\
\text { laryngospasm }\end{array}$ \\
\hline & mean $\pm \mathrm{SD}($ range $)$ & mean $\pm \mathrm{SD}($ range $)$ & mean $\pm \mathrm{SD}($ range $)$ & $p$ \\
\hline Age at evaluation, years & $56.9 \pm 11.4(29-77)$ & $56.2 \pm 12.1(37-77)$ & $58.1 \pm 10.7(29-72)$ & 0.423 \\
\hline Age at onset $^{\mathrm{a}}$, years & $43.5 \pm 12.2(14-66)$ & $42.9 \pm 12.3(14-63)$ & $44.4 \pm 12.5(23-66)$ & 0.943 \\
\hline $\begin{array}{l}\text { Disease duration } \\
\text { from onset }{ }^{\text {b }} \text { years }\end{array}$ & $13.1 \pm 10.2(2-59)$ & $12.8 \pm 11.2(2-59)$ & $13.4 \pm 8.8(3-33)$ & 0.667 \\
\hline $\begin{array}{l}\text { CAG-repeat length } \\
\text { in the AR gene, number }\end{array}$ & $48.5 \pm 3.9(40-60)$ & $48.2 \pm 3.2(40-54)$ & $49.1 \pm 4.9(43-60)$ & 0.853 \\
\hline Testosterone, ng/dL & $6.8 \pm 2.5(3.8-13.1)$ & $7.2 \pm 2.8(4.3-13.1)$ & $6.3 \pm 2.0(3.8-10.9)$ & 0.493 \\
\hline \multicolumn{5}{|l|}{ ALSFRS-R } \\
\hline Speech & $3.4 \pm 0.6(2-4)$ & $3.5 \pm 0.6(2-4)$ & $3.4 \pm 0.5(3-4)$ & 0.473 \\
\hline Salivation & $3.2 \pm 0.9(0-4)$ & $3.3 \pm 1.0(0-4)$ & $3.0 \pm 0.7(2-4)$ & 0.074 \\
\hline Swallowing & $3.4 \pm 0.6(2-4)$ & $3.5 \pm 0.6(2-4)$ & $3.2 \pm 0.5(2-4)$ & 0.108 \\
\hline Bulbar subscore $^{\mathrm{c}}$ & $10.0 \pm 1.6(5-12)$ & $10.3 \pm 1.7(5-12)$ & $9.6 \pm 1.3(8-12)$ & 0.054 \\
\hline Total & $40.0 \pm 4.6(26-48)$ & $40.5 \pm 4.9(26-48)$ & $39.4 \pm 4.3(34-48)$ & 0.229 \\
\hline \multicolumn{5}{|l|}{ Norris Scales } \\
\hline Limb Norris score & $53.0 \pm 8.1(27-62)$ & $53.5 \pm 8.4(27-62)$ & $52.3 \pm 7.9(39-62)$ & 0.961 \\
\hline Norris Bulbar score & $32.1 \pm 5.3(21-39)$ & $32.7 \pm 4.9(21-38)$ & $31.1 \pm 6.1(21-39)$ & 0.844 \\
\hline
\end{tabular}

3 Abbreviation: ALSFRS-R, revised amyotrophic lateral sclerosis functional rating

4 scale.

5 age at onset was defined as the age at which a patient became aware of muscular

6 weakness in any part of the body, including bulbar symptoms.

$7 \quad{ }^{\mathrm{b}}$ Disease duration from onset was defined as the period of time between the age at 8 onset and evaluation. 
$1{ }^{\mathrm{c}}$ The Bulbar subscore was calculated as the total number of items related to bulbar

2 signs consisting of speech, salivation, and swallowing.

3

4

5

6

7

8

9 
1 Table 3. Perceptual assessments of the 39 SBMA patients with and without 2 laryngospasm

\begin{tabular}{|c|c|c|c|c|}
\hline \multirow{3}{*}{ Parameters } & \multicolumn{4}{|c|}{ SBMA patients $(n=39)$} \\
\hline & $\begin{array}{l}\text { All } \\
(n=39)\end{array}$ & $\begin{array}{l}\text { Without } \\
\text { laryngospasm } \\
(\mathbf{n = 2 3})\end{array}$ & $\begin{array}{l}\text { With } \\
\text { laryngospasm } \\
(n=16)\end{array}$ & $\begin{array}{l}\text { With vs. } \\
\text { without } \\
\text { laryngospasm }\end{array}$ \\
\hline & mean $\pm \mathrm{SD}$ (range) & mean \pm SD $($ range $)$ & mean \pm SD $($ range $)$ & $p$ \\
\hline Hypernasality & $1.86 \pm 1.74(1-3.5)$ & $1.89 \pm 1.69(1-3.5)$ & $1.81 \pm 1.86(1-3)$ & 0.662 \\
\hline \multicolumn{5}{|l|}{$\begin{array}{l}\text { Hoarseness } \\
\text { (GRBAS scale) }\end{array}$} \\
\hline G & $1.14 \pm 0.84(0-3)$ & $1.13 \pm 0.71(0-2)$ & $1.17 \pm 1.03(0-3)$ & 0.921 \\
\hline $\mathbf{R}$ & $1.04 \pm 0.89(0-3)$ & $1.04 \pm 0.60(0-2)$ & $1.03 \pm 1.07(0-3)$ & 0.724 \\
\hline B & $0.35 \pm 0.54(0-2)$ & $0.39 \pm 0.60(0-2)$ & $0.28 \pm 0.45(0-1)$ & 0.746 \\
\hline $\mathbf{A}$ & $0.19 \pm 0.44(0-1.5)$ & $0.28 \pm 0.51(0-1.5)$ & $0.06 \pm 0.25(0-1)$ & 0.301 \\
\hline$S$ & $0.51 \pm 0.68(0-3)$ & $0.30 \pm 0.39(0-1)$ & $0.81 \pm 0.89(0-3)$ & 0.084 \\
\hline
\end{tabular}

3 Abbreviations: G, grade; R, roughness; B, breathiness; A, asthenia; S, strain.

4 Hypernasality scale: 1 , normal; 2, mild hypernasality; 3 , moderate hypernasality; and 4, severe hypernasality. GRBAS scale: 0 , normal; 1, mild deviance; 2 , moderate

6 deviance; and 3, severe deviance. 
1 Table 4. Acoustic analysis in SBMA using the MDVP analysis

\begin{tabular}{|c|c|c|c|}
\hline \multirow[b]{2}{*}{$\begin{array}{l}\text { Parameters } \\
\text { (Symbols) }\end{array}$} & \multicolumn{3}{|c|}{ SBMA $(n=37)$} \\
\hline & $\begin{array}{l}\text { Without } \\
\text { laryngospasm } \\
(\mathrm{n}=23) \\
\text { mean } \pm \mathrm{SD} \text { (range) }\end{array}$ & $\begin{array}{l}\text { With } \\
\text { laryngospasm } \\
(\mathrm{n}=14) \\
\text { mean } \pm \mathrm{SD} \text { (range) }\end{array}$ & $\begin{array}{l}\text { With vs. } \\
\text { without } \\
\text { laryngospasm } \\
p\end{array}$ \\
\hline \multicolumn{4}{|c|}{ Fundamental Frequency measurements } \\
\hline Fo $(\mathbf{H z})$ & $147.77 \pm 18.31(114.39-178.53)$ & $155.56 \pm 39.26(103.52-265.26)$ & 0.654 \\
\hline MFo (Hz) & $147.64 \pm 18.25(114.26-178.40)$ & $155.50 \pm 39.26(103.48-265.24)$ & 0.676 \\
\hline To $(\mathrm{ms})$ & $9.79 \pm 14.01(5.61-73.93)$ & $6.75 \pm 1.45(3.77-9.67)$ & 0.541 \\
\hline Fhi $(\mathbf{H z})$ & $159.80 \pm 21.19(127.07-198.38)$ & $167.73 \pm 37.55(121.17-272.64)$ & 0.676 \\
\hline Flo $(\mathrm{Hz})$ & $135.66 \pm 18.62(99.17-169.69)$ & $145.45 \pm 40.62(89.19-256.17)$ & 0.377 \\
\hline STD $(H z)$ & $3.59 \pm 3.38(1.10-18.02)$ & $2.64 \pm 0.90(1.28-5.00)$ & 0.526 \\
\hline PFR & $3.83 \pm 1.87(1.50-9.50)$ & $3.79 \pm 1.59(2.00-6.00)$ & 0.546 \\
\hline SEG & $99.95 \pm 0.15(99.50-100.00)$ & $99.96 \pm 0.14(99.50-100.00)$ & 0.914 \\
\hline PER & $440.85 \pm 55.36(341.50-533.50)$ & $464.76 \pm 128.23(309.00-795.50)$ & 0.654 \\
\hline \multicolumn{4}{|c|}{ Frequency perturbation measurements } \\
\hline Jita $(\mu \mathrm{m})$ & $103.83 \pm 63.37(19.51-247.12)$ & $60.28 \pm 35.85(16.85-131.51)$ & 0.039 \\
\hline Jitt $(\%)$ & $1.70 \pm 1.15(0.34-4.39)$ & $0.85 \pm 0.39(0.40-1.56)$ & 0.022 \\
\hline $\mathbf{R A P}(\%)$ & $0.91 \pm 0.63(0.16-2.54)$ & $0.48 \pm 0.24(0.17-0.89)$ & 0.039 \\
\hline PPQ (\%) & $0.92 \pm 0.59(0.20-2.28)$ & $0.51 \pm 0.25(0.22-0.97)$ & 0.042 \\
\hline $\operatorname{sPPQ}(\%)$ & $1.44 \pm 0.86(0.64-4.72)$ & $1.02 \pm 0.31(0.43-1.43)$ & 0.060 \\
\hline vFo $(\%)$ & $2.39 \pm 2.19(0.86-11.88)$ & $1.76 \pm 0.62(0.79-2.65)$ & 0.196 \\
\hline \multicolumn{4}{|c|}{ Amplitude perturbation measurements } \\
\hline ShdB (dB) & $0.40 \pm 0.16(0.13-0.77)$ & $0.30 \pm 0.15(0.11-0.72)$ & 0.050 \\
\hline $\operatorname{Shim}(\%)$ & $4.62 \pm 1.81(1.54-8.72)$ & $3.54 \pm 1.87(1.26-9.01)$ & 0.045 \\
\hline $\operatorname{APQ}(\%)$ & $3.89 \pm 1.31(1.72-6.35)$ & $3.26 \pm 1.53(1.07-7.05)$ & 0.125 \\
\hline SAPQ $(\%)$ & $5.94 \pm 2.04(3.04-10.92)$ & $5.68 \pm 2.00(2.47-9.31)$ & 0.511 \\
\hline $\mathbf{v A m}(\%)$ & $13.52 \pm 5.31(6.02-27.26)$ & $13.99 \pm 5.23(5.91-20.72)$ & 0.925 \\
\hline \multicolumn{4}{|c|}{ Voice break-related measurements } \\
\hline DVB $(\%)$ & $0.00 \pm 0.00(0.00-0.00)$ & $0.00 \pm 0.00(0.00-0.00)$ & 1.000 \\
\hline NVB & $0.00 \pm 0.00(0.00-0.00)$ & $0.00 \pm 0.00(0.00-0.00)$ & 1.000 \\
\hline \multicolumn{4}{|c|}{ Subharmonic component-related measurements } \\
\hline DSH $(\%)$ & $1.24 \pm 3.12(0.00-11.50)$ & $0.00 \pm 0.00(0.00-0.00)$ & 0.130 \\
\hline NSH & $1.22 \pm 3.06(0.00-11.50)$ & $0.00 \pm 0.00(0.00-0.00)$ & 0.130 \\
\hline \multicolumn{4}{|c|}{ Voice irregularity-related measurements } \\
\hline DUV (\%) & $0.31 \pm 1.07(0.00-5.00)$ & $0.54 \pm 1.28(0.00-4.50)$ & 0.495 \\
\hline NUV & $0.30 \pm 1.07(0.00-5.00)$ & $0.54 \pm 1.28(0.00-4.50)$ & 0.495 \\
\hline \multicolumn{4}{|c|}{ Noise-related measurements } \\
\hline NHR & $0.15 \pm 0.02(0.11-0.19)$ & $0.13 \pm 0.01(0.11-0.15)$ & 0.007 \\
\hline VTI & $0.04 \pm 0.01(0.02-0.07)$ & $0.03 \pm 0.01(0.01-0.05)$ & 0.039 \\
\hline SPI & $20.97 \pm 9.31(4.91-42.99)$ & $21.42 \pm 10.26(5.92-34.87)$ & 0.876 \\
\hline \multicolumn{4}{|c|}{ Tremor-related measurements } \\
\hline FTRI $(\%)$ & $0.58 \pm 0.24(0.24-1.34)$ & $0.56 \pm 0.28(0.15-0.98)$ & 0.511 \\
\hline ATRI (\%) & $4.64 \pm 2.74(0.63-12.80)$ & $5.13 \pm 2.16(1.02-7.71)$ & 0.391 \\
\hline Fftr $(\mathbf{H z})$ & $5.10 \pm 2.14(2.07-9.90)$ & $4.16 \pm 1.59(2.44-8.00)$ & 0.260 \\
\hline Fatr $(\mathrm{Hz})$ & $3.91 \pm 1.36(2.18-7.26)$ & $5.11 \pm 2.71(2.27-10.53)$ & 0.125 \\
\hline
\end{tabular}


1 Abbreviation: Fo, average fundamental frequency; MFo, mean fundamental

2 frequency; To, average pitch period; Fhi, highest fundamental frequency; Flo, lowest

3 fundamental frequency; STD, standard deviation of Fo; PFR, phonatory Fo-range in

4 semi-tones; SEG, number of segments computed; PER, total number detected pitch

5 periods; Jita, absolute jitter; Jitt, jitter percent; RAP, relative average perturbation;

6 PPQ; pitch perturbation quotient; sPPQ, smoothed pitch perturbation quotient; vFo,

7 fundamental frequency variation; ShdB, shimmer in dB; Shim, shimmer percent; APQ,

8 amplitude perturbation quotient; sAPQ, smoothed amplitude perturbation quotient;

9 vAm, peak-to-peak amplitude variation; DVB, degree of voice breaks; NVB, number

10 of voice breaks; DSH, degree of sub-harmonics; NSH, number of sub-harmonic

11 segments; DUV, degree of voiceless; NUV, number of unvoiced segments; NHR,

12 noise to harmonic ratio; VTI, voice turbulence index; SPI, soft phonation index; FTRI,

13 Fo-tremor intensity index; ATRI, amplitude tremor intensity index; Fftr, Fo-tremor

14 frequency; Fatr, amplitude tremor frequency. 
1 Table 5. Relationships between the acoustic parameters and clinical background

2 in the patients with SBMA

\begin{tabular}{|c|c|c|c|c|c|c|c|c|c|c|}
\hline \multirow{2}{*}{ Demographic } & \multicolumn{2}{|l|}{ Jita } & \multicolumn{2}{|l|}{ Jitt } & \multicolumn{2}{|l|}{ Shim } & \multicolumn{2}{|l|}{ VTI } & \multicolumn{2}{|l|}{ NHR } \\
\hline & $r$ & $p$ & $r$ & $p$ & $r$ & $p$ & $r$ & $p$ & $r$ & $p$ \\
\hline Age at evaluation, years & 0.059 & 0.727 & 0.012 & 0.942 & 0.218 & 0.194 & 0.146 & 0.388 & -0.108 & 0.526 \\
\hline $\begin{array}{l}\text { Disease duration from } \\
\text { onset }^{\mathrm{a}} \text {, years }\end{array}$ & 0.221 & 0.189 & 0.105 & 0.538 & 0.100 & 0.556 & -0.210 & 0.211 & -0.090 & 0.596 \\
\hline Testosterone, ng/dL & 0.061 & 0.721 & 0.119 & 0.484 & 0.093 & 0.584 & -0.069 & 0.683 & 0.069 & 0.683 \\
\hline $\begin{array}{l}\text { Frequency for the last } 6 \\
\text { months of laryngospasm }\end{array}$ & -0.319 & 0.055 & -0.346 & 0.036 & -0.328 & 0.047 & -0.305 & 0.066 & -0.460 & 0.004 \\
\hline ALSFRS-R & & & & & & & & & & \\
\hline Speech & -0.061 & 0.719 & 0.063 & 0.710 & 0.025 & 0.881 & 0.056 & 0.742 & -0.074 & 0.665 \\
\hline Salivation & 0.129 & 0.447 & 0.204 & 0.225 & 0.116 & 0.492 & 0.078 & 0.647 & 0.106 & 0.532 \\
\hline Swallowing & -0.062 & 0.714 & -0.010 & 0.953 & 0.029 & 0.863 & -0.111 & 0.514 & 0.224 & 0.182 \\
\hline Bulbar subscore $^{\mathrm{b}}$ & 0.029 & 0.863 & 0.136 & 0.421 & 0.105 & 0.535 & 0.034 & 0.841 & 0.115 & 0.497 \\
\hline Total & -0.075 & 0.659 & -0.028 & 0.870 & -0.005 & 0.977 & 0.028 & 0.867 & 0.047 & 0.783 \\
\hline Norris Scales & & & & & & & & & & \\
\hline Limb Norris score & -0.240 & 0.219 & -0.310 & 0.123 & -0.447 & 0.022 & -0.194 & 0.342 & -0.382 & 0.054 \\
\hline Norris Bulbar score & -0.048 & 0.810 & -0.027 & 0.879 & -0.149 & 0.467 & -0.255 & 0.208 & -0.084 & 0.682 \\
\hline
\end{tabular}

3 Abbreviations: ALSFRS-R, revised amyotrophic lateral sclerosis functional rating

4 scale; Jita, absolute jitter; Jitt, jitter percent; Shim, shimmer percent; VTI, voice

$5 \quad$ turbulence index; NHR, noise-to-harmonic ratio.

$6 \quad{ }^{\text {a }}$ Disease duration from onset was defined as the period of time between the age at 
1 onset and evaluation.

$2{ }^{\mathrm{b}}$ The bulbar subscore was calculated as the total number of items related to bulbar

3 signs consisting of speech, salivation, and swallowing. 
1 Table 6. Receiver operating characteristic curves for laryngospasm

\begin{tabular}{llllll}
\hline \multicolumn{1}{c}{ Variables } & Jita & Jitt & Shim & VTI & NHR \\
\hline Optimal cutoff point & 70.799 & 1.231 & 4.203 & 0.302 & 0.142 \\
Sensitivity, \% & 78.6 & 85.7 & 78.6 & 69.2 & 71.4 \\
Specificity, \% & 65.2 & 60.9 & 65.2 & 42.9 & 78.3 \\
AUC & 0.705 & 0.727 & 0.699 & 0.674 & 0.767 \\
$(\mathbf{9 5 \%}$ CI) & $(0.536-0.874)$ & $(0.565-0888)$ & $(0.518-0.879)$ & $(0.498-0.849)$ & $(0.615-0.919)$ \\
SE & 0.086 & 0.091 & 0.092 & 0.090 & 0.077 \\
$\boldsymbol{p}$ value & 0.017 & 0.022 & 0.045 & 0.079 & 0.007
\end{tabular}

2 Abbreviations: AUC, area under the curve; 95\% CI, 95\% confidence interval; SE,

3 standard error; Jita, absolute jitter; Jitt, jitter percent; Shim, shimmer percent; VTI,

4 voice turbulence index; NHR, noise-to-harmonic ratio.

5 The AUC, which goes from 0.5 to 1.0 , is a measurement value reflecting how good

6 the test is for distinguishing between patients with and without a specific symptom.

7 The closer the AUC is toward 1, the better the overall diagnostic performance of the

8 test (greater than 0.9, high accuracy; 0.7-0.9, moderate accuracy; and 0.5-0.7, low

9 accuracy). When the value of an MDVP parameter is lower than each optimal cut-off

10 point, laryngospasm is considered to be present. The actual receiver operating

11 characteristic curves are shown in Figure 1. 
Figure 1
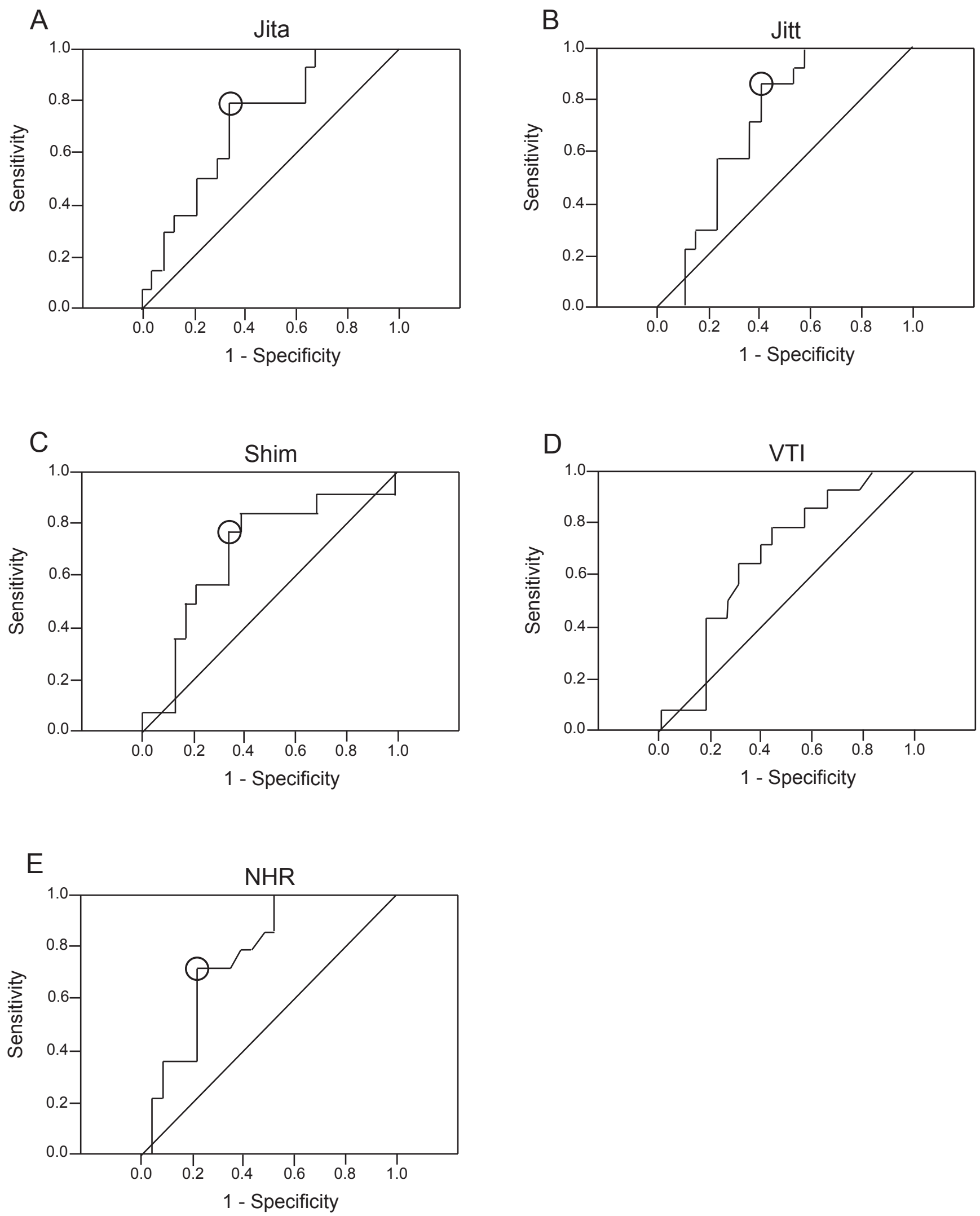